G. SUNOUCHI

KODAI MATH. J.

8 (1985), $1-4$

\title{
ON THE SUMMABILITY ALMOST EVERYWHERE OF THE MULTIPLE FOURIER SERIES AT THE CRITICAL INDEX
}

\author{
By GEN-ICHIRÔ SUNOUCHI
}

Recently Stein [5] shows the existence of an $H^{1}$ function on the $k$-torus, whose Fourier series is almost everywhere non-summable with respect to the Bochner-Riesz means of the critical index $(k-1) / 2$.

On the background of this example, he says as follows. In the case $k=1$, It is known that there exists an $H^{1}$ function whose Fourier series diverges almost everywhere (see [6], [7]). In the other direction the theorem of Carleson-HuntSjölin guarantees the convergence almost everywhere whenever $f \in L \log L$ $(\log \log L)$; see [3].

For the multiple Fourier series, S. Bochner pointed out that summability at the critical index $(k-1) / 2$ is the correct analogue of convergence for phenomena near $L$. In this sense Stein's example is a version in the case of general $k$. On the other hand, as another version he says that whenever $f \in L(\log L)^{2}$, the multiple Fourier series of $f$ is summable almost everywhere at the critical index $(k-1) / 2$. However this version is slightly different from the one dimensional case. The purpose of this note is to show that we can replace the last condition by $f \in L(\log L)(\log \log L)$.

Let $f(x)=f\left(x_{1}, x_{2}, \cdots, x_{k}\right) \in L$ on $Q_{k}:-\pi<x_{\imath} \leqq \pi \quad(i=1,2, \cdots, k)$ and its Fourier series be

where

$$
f(x) \sim \sum a_{n} e^{2 n \cdot x}
$$

$$
a_{n}=(2 \pi)^{-k} \int_{Q_{k}} f(x) e^{-2 n \cdot x} d x .
$$

Then the $\delta$-th Bochner-Riesz means of the series is

$$
\left(S_{R}^{\delta} f\right)(x)=\sum_{\mid n i<R}\left(1-|n|^{2} / R^{2}\right)^{\delta} a_{n} e^{\imath n \cdot x} .
$$

THEOREM. If $\int_{Q_{k}}|f(x)|\left(\log ^{+}|f(x)|\right)\left(\log ^{+} \log ^{+}|f(x)|\right) d x<\infty$, then,

$$
\lim _{R \rightarrow \infty}\left(S_{R}^{\alpha} f\right)(x)=f(x) \quad \text { a.e. }
$$

where $\alpha=(k-1) / 2(k>1)$.

Received January 30, 1984 
For the proof we generalize the index $\delta$ to complex number and set $\delta=\sigma+i \tau$. The estimate of Stein $[4$, p. 128] is, for $\sigma>\alpha$,

where

$$
\left(S_{*}^{\delta} f\right)(x) \leqq A_{\sigma} e^{\pi|z|}(\sigma-\alpha)^{-1} f^{*}(x)
$$

$$
\left(S_{*}^{\delta} f\right)(x)=\sup _{0<R<\infty}\left|\left(S_{R}^{\delta} f\right)(x)\right|
$$

and $f^{*}(x)$ is the spherical maximal function, that is

$$
\begin{gathered}
f^{*}(x)=\sup _{0<N<\infty} k N^{-k} \omega_{k}^{-1} \int_{|y|<N}|f(x+y)| d y, \\
\omega_{k}=2(\pi)^{k / 2} / \Gamma(k / 2) .
\end{gathered}
$$

The constant $A_{\sigma}$ remains bounded as $\sigma \rightarrow \alpha$. Since $f^{*}(x)$ is week type $(1,1)$, that is, $\mu$ being the Lebesgue measure,

$$
\mu\left\{x \mid f^{*}(x)>y\right\} \leqq \frac{A}{y}\|f\|_{1},
$$

where $A$ is a constant which depends on the dimension $k$ only, we have for $\sigma>\alpha$

$$
\mu\left\{x \mid\left(S_{*}^{\delta} f\right)(x)>y\right\} \leqq A_{\sigma} e^{\pi|\tau|}(\sigma-\alpha)^{-1} \frac{\|f\|_{1}}{y} .
$$

On the other hand it is routine for $f \in L^{2}$ to have

$$
\left\|\left(S_{*}^{\delta} f\right)(x)\right\|_{2} \leqq B_{\sigma} e^{\pi|\tau|}\|f\|_{z},
$$

for $\sigma>0$ and $B_{\sigma}=0\left(\sigma^{-1}\right)$ as $\sigma \rightarrow 0$. Hence we have naturally for $\sigma>0$

$$
\mu\left\{x \mid\left(S_{*}^{\delta} f\right)(x)>y\right\} \leqq\left(B_{\sigma} e^{\pi|\tau|} \frac{\|f\|_{2}}{y}\right)^{2}
$$

From (1) and (2) we shall prove the following proposition.

Proposition. Let $f(x) \in L^{p}\left(Q_{k}\right)$ and $\alpha$ be the critical index, then

$$
\mu\left\{x \mid\left(S_{*}^{\alpha} f\right)(x)>y\right\} \leqq\left(\frac{C}{p-1} \cdot \frac{\|f\|_{p}}{y}\right)^{p}
$$

for every $1<p<2$.

Proof. We apply to (1) and (2) the interpolation theorem on an analytic family of linear operators on $L(p, q)$ space (see [2]). The $L(p, q)$ space notation of (1) and (2) are

$$
\left\|\left(S_{*}^{\delta} f\right)(x)\right\|_{1, \infty}^{*} \leqq A_{\sigma} e^{\pi|\tau|}(\sigma-\alpha)^{-1}\|f\|_{1,1}^{*}
$$

for $\sigma>\alpha$, and

$$
\left\|\left(S_{*}^{\delta} f\right)(x)\right\|_{2, \infty}^{*} \leqq B_{\sigma} e^{\pi|\tau|}\|f\|_{2,2}^{*}
$$


for $\sigma>0$.

Let $R(x)$ be a measurable function on $Q_{k}$ such as $0 \leqq R(x) \leqq R_{0}<\infty$ and $\delta(z)$ $=\varepsilon_{0}(1-z)+\left(\alpha+\varepsilon_{1}\right) z$, where $\varepsilon_{0}, \varepsilon_{1}>0$ will be decided soon and we define an analytic family of linear operators

where $0 \leqq \operatorname{Re} z \leqq 1$.

$$
\left(T_{z} f\right)(x)=\left(S_{R(x)}^{\delta(z)} f\right)(x)
$$

For a given $p(1<p<2)$, we choose $\varepsilon_{0}=\alpha-(2-p) / 4 \geqq 1 / 2-(2-p) / 4=$ $p / 4>1 / 4>0$, and $\varepsilon_{1}=(p-1) / 2>0$. Set $(1-t) / 2+t / 1=1 / p$, that is $1 / p=(1+t) / 2$ $(0<t<1)$, then $\delta(t)=\varepsilon_{0}(1-t)+\left(\alpha+\varepsilon_{1}\right) t=\alpha$.

On the line $z=i y$, we have $\delta(i y)=\alpha-(2-p) / 4+i p y / 4$, that is

and

$$
\operatorname{Re} \delta(i y)=\varepsilon_{0}=\alpha-(2-p) / 4>1 / 4
$$

From (4) we have

$$
|\operatorname{Im} \delta(i y)|=p|y| / 4 \leqq|y| / 2 \text {. }
$$

$$
\left\|T_{\imath y} f\right\|_{2, \infty}^{*} \leqq\left\|\left(S_{*}^{\delta(i y)} f\right)(x)\right\|_{2, \infty} \leqq B_{\varepsilon_{0}} e^{\pi|y| / 2}\|f\|_{2,2}^{*} .
$$

Secondly on the line $z=1+i y$, we have $\delta(1+i y)=\alpha+(p-1) / 2+\imath p y / 4$, that is,

and

$$
\operatorname{Re} \delta(1+i y)=\alpha+(p-1) / 2>\alpha
$$

$$
|\operatorname{Im} \delta(1+i y)|=p|y| / 4 \leqq|y| / 2 \text {. }
$$

Hence from (3),

$$
\begin{aligned}
\left\|T_{1+\imath y} f\right\|_{1, \infty}^{*} & \leqq\left\|\left(S_{*}^{\delta(1+\imath y)} f\right)(x)\right\|_{1, \infty}^{*} \\
& \leqq A_{\alpha+\varepsilon_{1}}((p-1) / 2)^{-1} e^{\pi|y| / 2}\|f\|_{1,1}^{*} .
\end{aligned}
$$

Here we can write (5) and (6) to

$$
\begin{aligned}
& \left\|T_{\imath y} f\right\|_{2, \infty}^{*} \leqq K_{0}(y)\|f\|_{2,2}^{*} \\
& \left\|T_{1+\imath y} f\right\|_{1, \infty}^{*} \leqq K_{1}(y)\|f\|_{1,1}^{*}
\end{aligned}
$$

where $K_{0}(y) \leqq K_{0} e^{\pi|y| / 2}$ and $K_{1}(y) \leqq K_{1}(p-1)^{-1} e^{\pi|y| / 2}$. Therefore by the interpolation, we get

$$
\left\|\left(S_{*}^{\alpha} f\right)(x)\right\|_{p, \infty}^{*} \leqq A_{t}\|f\|_{p, p}^{*}
$$

where $A_{t}$ is given by

$$
\log A_{t}=\int_{-\infty}^{+\infty} \omega(1-t, y) \log K_{0}(y) d y+\int_{-\infty}^{\infty} \omega(t, y) \log K_{1}(y) d y,
$$

and $\omega(t, y)$ is the Poisson kernel for the strip $0 \leqq t \leqq 1,-\infty<y<\infty$. By the properties of Poisson kernel, we have 


$$
\begin{aligned}
& \omega(t, y) \geqq 0, \quad \int_{-\infty}^{\infty} \omega(1-t, y) d y \leqq 1, \quad \int_{-\infty}^{\infty} \omega(t, y) \leqq 1, \\
& \int_{-\infty}^{\infty} \omega(1-t, y)|y| d y \leqq K \text { and } \int_{-\infty}^{\infty} \omega(t ; y)|y| d y \leqq K .
\end{aligned}
$$

So we get $\log A_{t} \leqq \log C+\log (p-1)^{-1}$. Hence (9) becomes

$$
\left\|\left(S_{*}^{o} f\right)(x)\right\|_{p, \infty}^{*}<C(p-1)^{-1}\|f\|_{p, p}^{*},
$$

this means for $1<p<2$

$$
\mu\left\{x \mid\left(S_{*}^{a} f\right)(x)>y\right\}<\left(\frac{C}{p-1} \cdot \frac{\|f\|_{p}}{y}\right)^{p} .
$$

Thus we get the proposition.

From this proposition, we can derive our theorem by a lemma of CarlesonSjölin [3]. There is also a different type of proof of the lemma in [1, p. 481].

Remark. We reported this result before (Oct. 29, 1981) at the seminar on real analysis in Kanazawa (see Reports of the seminar on real analysis 1981 in Japanese). But the method of proof was more complicated. The proof of here was suggested by Professor M. Kaneko.

\section{LITERATURE}

[1] Calderon; C.P., Lacunary spherical means, Illinois Journ. Math., 23 (1979), 476-484.

[2] SAGHER, Y., On analytic families of operators, Israel Journ. Math., 7 (1969), 350-356.

[3] SJöLIN, P., An inequality of Paley and convergence a. e. of Walsh Fourier series, Arkiv för Mat., 7 (1968), 551-570.

[4] Stein, E. M., Localization and summability of multiple Fourier series, Acta Math., 100 (1958), 93-147.

[5] Stein, E. M., An $H^{1}$ function with non-summable Fourier expansion, Lecture notes in Math., No. 992, Springer, 1983, Harmonic analysis, Proc. Conf. Cortona, Italy, 193-200.

[6] Sunouchi, G., A Fourier series which belongs to the class $H$ diverges almost everywhere, Kodai Math. Seminar Rep., 5 (1953), 27-28.

[7] Zygmund, A., Trigonometric series, Cambridge Univ. Press, 1959.

Department of Technology

TAMAGAWA UNIVERSITY

MACHIDA, TOKYO 